\title{
Study of Single Scale Spatial Adaptive Filters, Diffusion Filters and Multi Scale Filters for Speckle Noise Suppression
}

\author{
Gagandeep Kaur \\ Department of Computer Science and Engineering \\ Guru Nanak Dev Engineering College \\ Ludhiana, Punjab, India
}

\author{
Bhupinder Singh \\ Assistant Professor \\ Lovely Professional University \\ Phagwara, Punjab, India
}

\begin{abstract}
Ultrasound imaging owing to its low cost and non-invasive nature is a widely accepted imaging technique. The image quality in ultrasound images is degraded by a special type of acoustic noise known as speckle. Speckle noise is a random presence of bright and dark spots which hinders human interpretation of images and computer assisted diagnostic techniques. The success of ultrasonic examination depends upon image quality; therefore, despeckling is necessary to improve visual quality for better diagnosis. Several speckle reduction methods are applied to the ultrasound images to suppress noise and preserve the useful diagnostic information. This paper presents a review of multi-scale filters (wavelets), single scale spatial adaptive filters (viz. Median, Wiener, Lee, Frost, Kuan, and Gamma MAP filter) and two Diffusion filters (viz. Anisotropic Diffusion filter and Speckle Reduction Anisotropic Diffusion filter)that are widely used for speckle reduction in biomedical ultrasound B-scan images.
\end{abstract}

\section{General Terms}

Digital Image Processing.

\section{Keywords}

Ultrasound imaging, speckle, single scale spatial adaptive filters, multi scale filters, anisotropic diffusion filters, shrinkage methods.

\section{INTRODUCTION}

Medical Ultrasound B-scan imaging is the most widely used imaging system in medical field for effective diagnosis of diseases [1]. It is used to visualize muscles and many other internal organs, their size, structure, and any pathological injuries with real time tomographic images. It does not use ionizing radiations, possessing noninvasive, portable and cost effective characteristics.

However ultrasound images are corrupted by speckle noise, subjected to imaging equipments and the external environment, when they are collected and transmitted. Ultrasonic speckle is an interference effect wherein, ultrasonic produced by ultrasonic probe is reflected by the body internal and external interface back to the probe [2-3]. The backscattered coherent waves having different phases undergo a constructive and destructive interference in a random fashion. The acquired images are thus degraded by a random granular pattern called speckle, which delays interpretation of the image content. Speckle noise reduces the spatial resolution of the imaging system thereby hinders the ability of a human observer to discriminate the fine details of diagnostic examination [4]. Therefore speckle noise suppression, by means of digital image processing, is a central pre-processing step for feature extraction, analysis and recognition from medical imagery measurements.

The paper is organized in the following way: Section 2 gives a model for speckle noise. Section 3 classifies the speckle reduction methods into two categories- single scale spatial adaptive filters and multi-scale filters. The subsections discuss the theoretical and mathematical forms of these methods. Section 4 briefly compares the performance of these filters. Paper concludes with Section 5.

\section{MODEL FOR SPECKLE NOISE}

Speckle is a spatially correlated multiplicative noise and expressed by Goodman [3] in a mathematical model for the analysis of speckle noise as [5]

$$
F(x, y)=G(x, y) \eta_{M}(x, y)+\eta_{A}(x, y)
$$

Where $F(x, y)$ is the real noisy image, $G(x, y)$ refers to the unknown noise free image, $\eta_{M}(x, y)$ and $\eta_{A}(x, y)$ are multiplicative and additive noise respectively. Since additive noise is considered to be lower than multiplicative noise, signal dependent noise model for speckle specification in Ultrasound images is generally modeled as

$$
F(x, y)=G(x, y) \eta_{M}(x, y)
$$

\section{SPECKLE FILTERING TECHNIQUES}

Several techniques to remove speckle have been proposed by researchers [6]. These speckle reduction filters can be classified into two categories viz. compounding method and post acquisition method [7].

Compounding methods [8] are based on averaging of multiple images of the same scan plane, where images are obtained by varying transducer frequency and/or view angle to achieve independent or partially uncorrelated speckle pattern. These methods can improve the target detectability but need hardware modifications and suffer from degradation of spatial resolution and increased system complexity.

On the other hand, the post acquisition methods do not need hardware modification. These methods improve the image details and reduce the speckle noise considerably via algorithm implementations. These methods are further categorized into single scale spatial adaptive filters and multiscale filters.

\subsection{Single Scale Spatial Adaptive Filtering}

This approach consists of moving a square window called kernel over each pixel in the image and establishing a statistical relationship, such as local mean or local variance, between the center pixel and its surrounding pixels. The value 
of the central pixel is replaced with the calculated value. This approach improves the smoothing effect in homogenous regions where speckle is fully developed and the effect lessens in other regions to preserve useful details of the image [7]. The typical size of filter window must be odd and varies from $3 \times 3$ to $33 \times 33$. Generally a $3 \times 3$ or $7 \times 7$ window yields good results [9].

The single scale spatial adaptive filters involve three main steps:

1. Computation of local statistics like local mean or variance.

2. Region growing procedure where a pixel is grouped into homogenous regions using certain homogeneity criteria like gray level, texture, local statistics, color similarities.

3. Application of smoothening operator wherein mean or median is applied on homogenous regions.

The speckle reduction filters in this category are mean, median, Lee, Kuan, Frost, Enhanced Frost, Gamma MAP, Diffusion filters. The following section presents theoretical and mathematical description of these filters.

\subsubsection{Median Filter}

The Median filter [10] is a spatial non-linear filter which can preserve edges and other spatial details contained in the image. It finds application, when the noise pattern consists of strong spike like components. The median filter replaces the middle pixel value in a moving window with the median of the gray values of pixels in a specific neighborhood. The spatial extent of the neighborhood and the number of pixels involved in the median calculation determine the noise reducing effect of median filter. The extra computation time needed to sort the intensity value of each set is a great limitation of median filter.

\subsubsection{Wiener Filter}

The Wiener filter [11] also known as Least Mean Square filter can restore images degraded with blur as well as noise. The statistical parameter used to perform smoothing of the image is local image variance. The smoothing effect is little where the variance is large and vice-versa. It preserves the edges and other high frequency details of the image.

Mathematically, Wiener filter is given by the following expression:

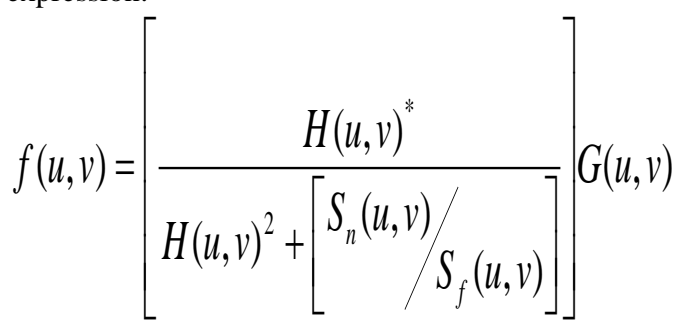

Where $\mathrm{H}(\mathrm{u}, \mathrm{v})$ is the degradation function $(*$ indicates complex conjugate) and $\mathrm{G}(\mathrm{u}, \mathrm{v})$ is the degraded image. Functions $S_{f}(u, v)$ and $S_{n}(u, v)$ are power spectra of the original image and the noise respectively.

\subsubsection{Lee Filter}

The Lee filter [12] assumes a Gaussian (normal) distribution for the noise values and uses a minimum mean square error to estimate the true strength of the center pixel in the filter window. This estimate is calculated from the measured value of that pixel, the local mean brightness of all pixels in the window, and a gain factor calculated from the local variance and the noise standard deviation. For uniform areas, the Lee filter calculates an output value close to the local mean, and a value close to the original input value in higher contrast regions. The Lee filter produces the speckle free image governed by the relationship given below

$U(x, y)=I(x, y) W(x, y)+I^{\prime}(x, y)(1-W(x, y))$

Where $I^{\prime}$ is the mean value of the intensity within the filter window, and $W(x, y)$ is the adaptive filter coefficient calculated using the following formula.

$$
W(x, y)=1-\frac{C_{B}^{2}}{C_{I}^{2}+C_{B}^{2}}
$$

Where $C_{I}$ is the coefficient of variation of the noised image and $C_{B}$ is the coefficient of variation of the noise. In general, the value $W(x, y)$ approaches zero in uniform areas, i.e., it approaches unity at edges which results in little modification of pixel values near edges.

\subsubsection{Frost Filter}

The Frost filter [13] is an adaptive filter which assumes a negative exponential distribution for speckle noise and uses local image statistics in the filtering process. The Frost filter computes a weighted average of the cell values in the filter window, where the weight for each pixel is determined from the local statistics to minimize the mean square error of the signal estimate. The weighting factor decreases with the distance from the center pixel and is weighed more heavily as the variance in filter window increases. The parameters in the Frost filter are adjusted according to the local variance in each area. The filtering process can cause extensive smoothing in areas where variance is low. On the other hand, edges are retained and little smoothing takes place in the areas where variance is high. The response of the filter is given by

$$
W(x, y)=\frac{\left(\sum P_{n} * W F_{n}\right)}{\sum W F_{n}}
$$

Where

$$
W F_{n}=\exp \left(-D F *\left(\frac{S D}{L M}\right)^{2} * T\right)
$$

$P_{n}$ represent the image pixels in the filter window. $D F$ is the damping factor, which determines the extent of the potential damping for the image. $S D$ is the standard deviation of the filter window. $L M$ is the local mean $T$ is the absolute value of the pixel distance between the centre pixel to its surrounding pixels in the filter window.

\subsubsection{Kuan Filter}

The Kuan [14] filter is a local linear minimum square error filter which models the multiplicative model of speckle noise into an additive linear form. The Kuan filter assumes that the mean and variance of pixel of interest is equal to the local mean and variance of all pixels within the moving window. The Kuan filter can be described as:-

$$
\hat{R}(t)=\bar{I}(t)+[I(t)-\bar{I}(t)] \times W(t)
$$

Where $\mathrm{W}$ is the weighting function ranging between 0 for flat regions and 1 for regions with high signal activity, $\bar{I}$ is the average of pixels in a moving window and $\hat{R}(i, j)$ is the output of the filter. The weighting function of the Kuan filter is defined as 
$W(t)=\frac{1-C_{u}^{2} / C_{I}^{2}(t)}{1+C_{u}^{2}}$

where

$$
C_{u}=\frac{\sigma_{u}}{\bar{u}} \text { and } C_{I}=\frac{\sigma_{I}}{\bar{I}}
$$

are the coefficients of variations of the noise $u$ and the image I.

\subsubsection{Gamma MAP Filter}

A speckle reduction filter based on Maximum A Posteriori (MAP) [15] approach, requires a prior knowledge of probability density function (pdf) of the image. This filter minimizes the loss of texture information of agricultural lands, forested areas, and oceans by assuming the images to be gamma distributed.

It uses coefficient of variation and contrast ratio whose theoretical pdf determines the smoothing process. The filtered pixel value depends upon the comparison between the local coefficient of variation $C_{I}$ in filter window of defined size, the speckle coefficient of variation using equivalent number of looks $C_{L}$ and $C_{\max }$ the upper speckle coefficient of variation of the image.

If $C_{I}$ falls between $C_{L}$ and $C_{\max }$ the value of filtered pixel in a defined window size is given as

$U\left(x^{\prime}, y^{\prime}\right)=\frac{(\alpha-L-1) I^{\prime}\left(x^{\prime}, y^{\prime}\right)+\sqrt{I^{\prime 2}\left(x^{\prime}, y^{\prime}\right)(\alpha-L-1)+4 \alpha L I^{\prime}\left(x^{\prime}, y^{\prime}\right)}}{2 \alpha}$

Where

$I\left(x^{\prime}, y^{\prime}\right)$ is the mean intensity value within the window. $\alpha$ is the weighting function given as

$$
\alpha=\frac{1+C_{L}^{2}}{C_{I}^{2}-C_{L}^{2}}
$$

$\mathrm{L}$ is the number of looks

$C_{I}$ is the speckle coefficient of variation of the filter window and

$$
C_{I}=S / I
$$

$C_{L}$ is the speckle coefficient of variation using equivalent number of looks

$$
C_{L}=1 / \sqrt{L}
$$

$C_{\text {max }}$ is the upper speckle coefficient of variation of the image

$$
C_{\max }=\sqrt{2} * C_{L}
$$

$\mathrm{S}$ is the standard deviation in filter window

For $C_{I}$ smaller than $C_{L}, U\left(x^{\prime}, y^{\prime}\right)$ equals $I^{\prime}\left(x^{\prime}, y^{\prime}\right)$ and if $C_{I}$ is greater than $C_{\max }$ then the filtered pixel $U\left(x^{\prime}, y^{\prime}\right)$ has the same value as the center pixel in the filter

\subsection{Diffusion Filters}

Diffusion filters proposed by Persona and Malik [17] remove the speckle by modifying the image via solving Partial Differential Equation (PDE). It simultaneously removes the speckle and enhances the edges. An edge detection function controls the strength and the direction of the diffusion. A simple isotropic diffusion equation may be given as: $\frac{\partial I(i, j, t)}{\partial t}=\operatorname{div}(c \nabla I)$

where $I(i, j, t)=0$ is an image in the continuous domain, $t$ is an artificial time parameter, when $\mathrm{t}=0$, its $I$ is original image, as $\mathrm{t}$ increases, image $I$ becomes more smooth. "c" is the diffusion constant, and $\nabla I$ is the image gradient. This linear isotropic diffusion equation is equivalent to Gaussian filtering. There are two diffusion filters namely, Anisotropic Diffusion (AD) and Speckle Reducing Anisotropic Diffusion (SRAD).

\subsubsection{Anisotropic Diffusion}

Anisotropic Diffusion [18] is an efficient, non-linear technique which simultaneously performs contrast enhancement and noise reduction. The filter encourages smoothening within the region as compared to the edges. Persona and Malik replaced the classical isotropic diffusion equation by anisotropic diffusion method for smoothing image on a continuous domain. The diffusion is described by

$$
\begin{aligned}
& \frac{\partial I}{\partial t}=\operatorname{div}[c(\|\nabla I\|) . \nabla I] \\
& I(t=0)=I_{0}
\end{aligned}
$$

Where, div is the divergence operator, $\|\nabla I\|$ is the gradient magnitude of the image $I, c(\|\nabla I\|)$ is the diffusion coefficient or the diffusivity function and $I_{0}$ is the original image. If the function $\mathrm{c}(\|\nabla I\|)$ is constant for all image locations the diffusivity function, c $(\|\nabla I\|)$, is a monotonically decreasing function of the gradient magnitude. Persona and Malik suggested two different edge stopping $\mathrm{c}(\mathrm{x})$ functions in their anisotropic diffusion equation

$$
\begin{aligned}
& c(\nabla I)=\frac{1}{1+\left\{\frac{(|\nabla I|)}{K}\right\}^{2}} \\
& c(\nabla I)=e^{\left(-\frac{(\nabla I \mid)}{K}\right)^{2}}
\end{aligned}
$$

Where, $\mathrm{K}$ is edge magnitude parameter.

A step discontinuity gradient magnitude is used to find the edges. If $\|\nabla I\|>>K$ then $c(\|\nabla I\| \rightarrow 0)$, an all pass filter is used; if $\|\nabla I\|<<K$, then $c(\|\nabla I\|) \rightarrow 1$, isotropic diffusion is achieved. The edge stopping function in equation (17) can remove noise in large area efficiently, because its diffusion is in inverse proportion to gradient. But this function cannot preserve edge information. The exponent edge stopping function in (18) can retain edges.

\subsubsection{Speckle Reducing Anisotropic Diffusion (SRAD)}

SRAD [19] is an edge preserving diffusion method where diffusion constant is proposed in terms of the "instantaneous coefficient of variation" as a function of the local gradient magnitude and Laplacian operators. The function exhibits high values at edges and produces low values in homogenous 
regions. Thus it ensures the mean preserving behavior in the homogenous regions and edge preserving and edge enhancing at the edges. The PDE based speckle removal approach generates of an image scale space i.e. a set of filtered images that vary from fine to coarse without bias due to filter window size and shape. Given an intensity image $I_{0}(x, y)$ having finite power and no zero values over the image support $\Omega$, the output image $\mathrm{I}(\mathrm{x}, \mathrm{y} ; \mathrm{t})$ is evolved according to the following PDE:

$$
\left\{\begin{array}{l}
\partial I(x, y ; t) / \partial t=\operatorname{div}[c(q) \Delta I(x, y ; t)] \\
I(x, y ; 0)=I_{0}(x, y),(\partial I(x, y ; t) / \partial \vec{n}) \mid \partial \Omega=0
\end{array}\right.
$$

where $\partial \Omega$ denotes the border of $\Omega, \vec{n}$ is the outer normal to the $\partial \Omega$ and

$$
c(q)=\frac{1}{1+\left[q^{2}(x, y ; t)-q_{0}^{2}(t)\right] /\left[q_{0}^{2}(t)\left(1+q_{0}^{2}(t)\right)\right]}
$$

Or

$$
c(q)=\exp \left\{-\left[q^{2}(x, y ; t)-q_{0}^{2}(t)\right] /\left[q_{0}^{2}(t)\left(1+q_{0}^{2}(t)\right)\right]\right\}
$$

In (18) and (19), $q(x, y ; t)$ is the instantaneous coefficient of variation determined by

$$
q(x, y ; t)=\sqrt{\frac{\left(\frac{1}{2}\right)\left(\frac{|\Delta I|}{I}\right)^{2}-\left(\frac{1}{4^{2}}\right)\left(\frac{\Delta^{2} I}{I}\right)^{2}}{\left[1+\left(\frac{1}{4}\right)\left(\frac{\Delta^{2} I}{I}\right)\right]^{2}}}
$$

And $q_{0}$ is the speckle scale function. In the SRAD, the instantaneous coefficient of variation $q(x, y ; t)$ serves as the edge detector in speckled imagery. The function exhibits high values at the edge or on high contrast features and produces low values in homogenous regions. The modification reflects encouraging isotropic diffusion in homogenous regions of the image where $q(x, y ; t)$ fluctuates around $q_{0}(t)$. The speckle scale function $q_{0}(t)$ effectively controls the amount of smoothing applied to the image by SRAD. It is estimated using

$$
q_{0}(t)=\frac{\sqrt{\operatorname{var}[z(t)]}}{z(t)}
$$

where var $[\mathrm{z}(\mathrm{t})]$ and $\overline{z(t)}$ are the intensity variance and mean over a homogenous area at $t$, respectively.

\subsection{Multi-scale Filtering}

Multi-scale analysis proposed by Mallat for image processing applications, can be viewed as a successive approximation or successive refinement of a signal and is closely related with the wavelet transform [20][21]. The approximations are the high-scale, low-frequency components of the signal whereas the details are the low-scale, high-frequency components. The DWT (Discrete Wavelet Transform) separates an image into a lower resolution approximation image (LL) as well as horizontal (HL), vertical (LH) and diagonal $(\mathrm{HH})$ detail components.

Wavelet denoising attempts to remove noise present in the signal while preserving the signal characteristics, regardless of its frequency content. Multi-scale wavelet transform utilizes the "focus" capability in the filtering method which means that a small number of wavelet coefficients are better than other wavelet coefficients. They contain the most of the signals which can be separated using wavelet threshold procedure.

The multi-scale analysis of wavelet based speckle reduction process usually includes (1) logarithmic transformation (2) Discrete wavelet transformation (3-level decompositions) (3) Thresholding the wavelet coefficients (Threshold may be universal or sub band adaptive) (4) inverse discrete wavelet transform and (5) exponential transformation.

Thresholding distinguishes between the coefficients due to noise and the ones consisting of important signal information. There are two general categories of thresholding, hardthresholding and soft-thresholding [22]. Hard-thresholding function retains all coefficients whose magnitude is greater than the selected threshold value, $t$, and the others with magnitudes smaller than $t$ are set to zero. Soft-thresholding shrinks the coefficients greater than the threshold towards zero after comparing them to a threshold value. Softthresholding is preferred over hard-thresholding, for the softthresholding method yields more visually pleasant images over hard thresholding which yields abrupt artifacts in the recovered image. The choice of a threshold plays a major role in the removal of noise in images .A small threshold may yield a result close to the input, but it may still be noisy. Large threshold alternatively, produces signal with large number of zero coefficients which leads to a smooth signal. So much attention must be paid to select optimal threshold.

Speckle reduction through wavelet transform based on Bayesian approach by means of the statistical models of free signal and noise is suggested by Achim et.al [23], Thitimajshima.P et.al [24]. Wavelet-based denoising using Hidden Markov Models (HMM) [25] models are efficient in capturing inter-scale dependencies and has been quite successful. The various shrinkage methods, as discussed below, are based on estimation of statistical and probabilistic parameters required for shrinkage.

\subsubsection{VisuShrink}

VisuShrink [26] is a thresholding method proposed by Dohono and Johnstone. It uses a threshold value that is proportional to the standard deviation of the noise. It can be viewed as general-purpose threshold selector that exhibits near optimal minimax error property and is found to yield a highly smoothed estimate. This threshold is given by:

$$
T_{U}=\sigma_{n} \sqrt{2 \log L}
$$

Where, $\sigma_{n}^{2}$ is the noise variance of AWGN and L is the total number of pixels in an image. $T_{U}$ tends to be high for large values of L, killing many signal coefficients along with the noise. It cannot remove speckle noise as it deals with an additive noise only. VisuShrink follows the global 
thresholding scheme where there is a single value of threshold applied globally to all the wavelet coefficients.

\subsubsection{SureShrink}

SureShrink [27] is an adaptive thresholding method and is based on Stein's Unbiased Risk Estimator (SURE). The thresholding employed here is adaptive, i.e., a threshold level is assigned to each dyadic resolution level by the principle of minimizing the Stein's Unbiased Risk Estimator for threshold estimates. The threshold $\mathrm{T}_{\mathrm{SURE}}$ employed for denoising is expressed as:

$T_{\text {sure }}=\arg \min _{T K}\left(\operatorname{SURE}\left(T_{h} ; Y\right)\right)$

$\left(\operatorname{SURE}\left(T_{h} ; Y\right)\right)$ is defined by:

$\operatorname{SURE}\left(T_{h}: Y\right)=\sigma^{2}-\frac{1}{L} \times\left[2 \sigma_{n}^{2},\left\{i:\left|Y_{i}\right| \leq T_{h}-\sum \min \left(\left|Y_{i}\right|, T_{h}\right)^{2}\right\}\right]$

Where, $\sigma_{n}^{2}$ is the noise variance of AWGN; L is the total number of coefficients in a particular sub-band; $Y_{i}$ is a wavelet coefficient in the particular sub-band. SureShrink follows the soft thresholding rule. It is smoothness adaptive, which means that if the unknown function contains abrupt changes or boundaries in the image, the reconstructed image also does.

\subsubsection{BayesShrink}

BayesShrink [28] uses an adaptive data-driven threshold for image denoising. This method is based on the Bayesian mathematical framework. The wavelet coefficients of a natural image are modeled by a Generalized Gaussian Distribution (GGD). Thus, a threshold is derived in a Bayesian framework as:

$$
T_{B}=\left(\frac{\hat{\sigma}_{n}^{2}}{\hat{\sigma}_{F}}\right)
$$

Where, $\hat{\sigma}_{n}{ }^{2}$ is the estimated noise variance of AWGN by robust median estimator and $\sigma_{F}$ is the estimated signal standard deviation in wavelet-domain.

\subsubsection{NeighShrink}

Chen et al. incorporated neighboring coefficients in the wavelet-domain image thresholding scheme [29]. The method, NeighShrink, thresholds the wavelet coefficients according to the magnitude of the squared sum of all the wavelet coefficients, i.e., the local energy, within the neighborhood window. The neighborhood window size may be $3 \times 3,5 \times 5,7 \times 7,9 \times 9$, etc. The shrinkage function for NeighShrink of any arbitrary $3 \times 3$ window centered at $(i, j)$ is expressed as:

$$
\Gamma_{i j}=\left(1-\frac{T_{U}^{2}}{S_{i j}^{2}}\right)_{+}
$$

Where, $\mathrm{T}_{\mathrm{U}}$ is the universal threshold and $S_{i j}{ }^{2}$ is the squared sum of all wavelet coefficients in the respective $3 \times 3$ window given by

$$
S_{i j}^{2}=\sum_{m=i-1}^{i+1} \sum_{n=j-1}^{j+1} w_{m, n}^{2}
$$

Here, + sign at the end of the formula means to keep the positive values while setting it to zero when it is negative. The

estimated center wavelet coefficient $F_{i j}$ is then calculated from its noisy counterpart $\mathrm{w}_{\mathrm{ij}}$ as:

$$
\hat{F}_{i j}=\Gamma_{i j} w_{i j}
$$

\section{CONCLUSION}

The existing despeckling techniques for ultrasound images can preserve image features as well as edges. But they have constraints regarding resolution degradation. The filters operate by smoothing over a fixed window; large window size reduces the resolution of the algorithm, and produces artifacts around the object. Wavelet transform performs better than the standard speckle filters and is best suited due to its properties like sparsity, multiresolution and multi-scale nature. Thresholding techniques are simplest to implement with discrete wavelets. The choice of despeckling filters and speckle model plays an important role in the design of despeckling methods, and it varies from application to application. The paper discussed most commonly employed models and filters along with their pros and cons.

\section{REFERENCES}

[1] Chris Guy, and Dominic Ffytche, "An Introduction to the principles of medical imaging," Revised edition, Imperial College Press, pp.267- 294, 2005.

[2] Robert F. Wagner, Stephen W. Smith, John M. Sandrik, and Hector Lopez, "Statistics of Speckle in Ultrasound B- Scans," IEEE Trans. on Sonics and Ultrasonics, Vol. 30, no. 3, pp.156-163, May 1983.

[3] J.W. Goodman, "Some Fundamental Properties of Speckle," Journal of Optical Society of America, Vol.66, No.11, pp.1145-1150, November 1976.

[4] Christoph B. Burckhardt, "Speckle in Ultrasound BMode Scans," IEEE Trans. on Sonics and Ultrasonics, Vol.25, No.1, pp.1-6, 1978.

[5] Anil K. Jain, "Fundamentals of Digital Image Processing," first edition, Prentice-Hall, Inc, 1989.

[6] Stian Solbo and Torbjorn Eltoft, "Homomorphic Wavelet-Based Statistical Despeckling of SAR Images," IEEE Trans. on Geoscience and Remote Sensing, Vol. 42, No. 4, pp.711-721, April 2004.

[7] S.Kalaivani Narayanan and R.S.D. Wahidabanu, "A View on Despeckling in ultrasound imaging," International Journal of Signal Processing.Image Processing and Pattern Recognition, Vol.2, No.3, September 2009. 
[8] M.O'Donnel and S.D Silverstein, "Optimum displacement for compound image generation in medical Ultrasound,'IEEE Trans Ultrason. Ferroelect.Freq.Contr., vol.35,no.4,pp.470-476,July.1988

[9] Hong Sern Tan, “ Denoising of Noise Speckle in Radar Image”, Oct. 2001

[10] T.Loupas, W.N. Mcdicken and P.L Allen, "An adoptive weighted median filter speckle suppression in medical ultrasound images," IEEE Trans. Circuits Sys., Vol.36, pp. 129-135, 1989.

[11] T. Kailath, "Equations of Wiener-Hopf type in filtering theory and related applications," in Norbert Wiener: Collected Works vol. III, P.Masani, Ed. Cambridge, MA: MIT Press, 1976, pp. 63-94.

[12] J. S. Lee, "Digital Image Enhancement and Noise Filtering by Use of Local Statistics," IEEE Trans. on Pattern Analysis and Machine Intelligence, Vol. PAMI2, No. 2, pp.165-168, March 1980.

[13] V.S.Frost, J.A.Stiles, K.S.Shanmugam, J.C.Holtzman, "A model for radar image \& its application to Adaptive digital filtering for multiplicative noise", IEEE Transaction on pattern analysis and machine intelligence, Vol.PMAI 4, pp.175-166, 1982.

[14] D. T. Kuan, A. A. Sawchuk, T.C. Strand, and P. Chavel, "Adaptive restoration of images with speckle," IEEE Trans. on Acoustics, Speech and Signal Processing, Vol. ASSP-35, No.3, pp.373-383, March1987.

[15] Lopes.A, Nesry.E, Touzi.R, Laur.H., "Maximum A Posteriori speckle filtering and first order texture models in SAR images". Proceedings of IGARSS' 90, May 1990, vol. 3 (Maryland: IGARSS), pp. 2409-2412, 1990.

[16] Jappreet Kaur, Jasdeep Kaur, Manpreet Kaur.”Survey of despeckling techniques for medical images." International Journal of Computer Technology and Application , vol 2 (4),Pages 1003-1007,Jult-August 2011

[17] P.Perona and J.Malik,"Scale space and edge detection using anisotropic diffusinon,'IEEE Trans. Pattern Anal.Machni Intell.,vol12,pp.629-639,1990

[18] P. Perona and J. Malik, "Scale space and edge detection using anisotropic diffusion," IEEE Trans. on Pattern Analysis and Machine Intelligence, Vol.12, No.7, pp. 629-639, JULY 1990.
[19] Y.J.Yu \& S.T.Action,"Speckle reducing anisotropic diffusion,"

Trans.Imag.Process.,vol.11,no.11pp.1260-1270,Nov.2002.

[20] S.G. Mallat, "A theory for multiresolution signal decomposition: the wavelet representation," IEEE Trans. On Pattern Analysis and Machine Intelligence, Vol.11, No.7, pp.674-693, July 1989.

[21] S.G. Mallat, "Multifrequency channel decomposition of images and wavelets models," IEEE Trans. On Acoustics, speech, and Signal Processing, Vol.37, No.12, pp.2091-2110, December 1989

[22] Q. Pan "Two denoising methods by wavelet transform, " IEEE Trans. Signal Processing, vol. 47, pp. 3401-3406, Dec. 1999.

[23] Achim Bezerianos A. and Tsakalides.P, "Novel Bayesian Multiscale for Speckle Removal in Medical Ultrasound Images", IEEE Transactions. Medical Imaging Journal, 20[8], pp. 772-783, 2001.

[24] Thitimajshima.P, Rangsanseri.Y, and Rakprathanporn.P, "A Simple SAR Speckle Reduction by Wavelet Thresholding", Proceedings of the 19th Asian Conference on Remote Sensing ACRS98, pp. P-14-1P-14-5, 1998

[25] Crouse. M. S., R. D. Nowak, and R. G. Baraniuk. “ Wavelet based statistical signal Processing using Markov models", IEEE Trans. Signal Processing, Vol. 46, no.4 pp. 886- 902, 1998

[26] D. L. Donoho and I. M. Johnstone, "Ideal spatial adaptation via wavelet shrinkage," Biometrika, vol. 81, pp. 425-455, 1994.

[27] D. L. Donoho and I. M. Johnstone , "Adapting to unknown smoothness via wavelet shrinkage" J. Amer. Statist. Assoc., vol. 90, pp. 1200-1224, December 1995.

[28] S. Chang, B. Yu, M. Vetterli, "Adaptive wavelet thresholding for image denoising and compression", IEEE Transactions on Image Processing, vol. 9, no. 9, pp. 1532-1546, 2000

[29] G.Y. Chen, T.D. Bui, A Krzyzak, "Image denoising using neighboring wavelet coefficients", proceedings of IEEE international conference on Acoustics, speech and signal processing '04, pp. 917-920, 2004. 